\title{
Modulation of lysine acetylation-stimulated repressive activity by Erk2-mediated phosphorylation of RIP140 in adipocyte differentiation
}

\author{
Ping-Chih Ho, Pawan Gupta, Yao-Chen Tsui, Sung Gil Ha, MD Mostaqul Huq, and Li-Na Wei \\ Department of Pharmacology, University of Minnesota Medical School, Minneapolis, MN 55455, \\ USA
}

\begin{abstract}
Receptor-interacting protein 140 is a co-regulator for many transcription factors. Previous mass spectrometry studies showed that either phosphorylation or lysine acetylation of RIP140 directly enhanced its trans-repressive activity. In this study, we first identified p300 as a specific lysine acetyltransferase, and extracellular-signal-related kinase 2 (Erk2) as a specific kinase for threonine phosphorylation, of RIP140 in vivo. We further determined two specific acetylated lysine residues $\left(\right.$ Lys ${ }^{158} / \mathrm{Lys}^{287}$ ) and phosphorylated threonine residues $\left(\mathrm{Thr}^{202} / \mathrm{Thr}^{207}\right.$ ) that were critical for its gene repressive activity. We then delineated signal transduction from Erk2-mediated phosphorylation of RIP140 that enhanced its recruiting p300 for subsequent lysine acetylation, and demonstrated the kinetics of activation of this signal transduction pathway in differentiating adipocytes. Finally, the physiological significance of this cell signal transduction pathway was illustrated in rescuing experiments where the defect in fat accumulation of RIP140-null cultures was rescued by reexpressing the wild type RIP140 or its phospho-mimetic mutant, but not its acetylation deficient mutant. These results demonstrate the signal transduction pathway, initiated from Erk2 activation for specific threonine phosphorylation, followed by p300 recruitment for lysine acetylation, which ultimately enhances the gene-repressive activity of RIP140 and its functional role in fat accumulation in differentiated adipoctyes.
\end{abstract}

\section{Keywords}

Receptor interacting protein 140; post-translational modification; p300; Extracellular-signal-related kinase 2; Acetylation; Phosphorylation; lipid metabolism

\section{Introduction}

Receptor-interacting protein 140 (RIP140), or NRIP1 (Nuclear Receptor Interacting Protein 1 ), is known as a versatile transcriptional co-repressor for all the tested nuclear receptors and many transcription factors. The transcription regulatory activity of RIP140 is mediated through its four autonomously repressive domains $[1,2]$ that recruit components of other co-repressive machineries such as histone deacetylases (HDACs) and CtBP [3]. By using in vitro assembled

\footnotetext{
*Correspondence: Li-Na Wei, Department of Pharmacology, University of Minnesota Medical School, 6-120 Jackson Hall, 321 Church Street SE, Minneapolis, MN 55455-0217, USA., Tel: 1-612-625-9402, Fax: 612-625-8408, E-mail: weixx009@umn.edu.

Publisher's Disclaimer: This is a PDF file of an unedited manuscript that has been accepted for publication. As a service to our customers we are providing this early version of the manuscript. The manuscript will undergo copyediting, typesetting, and review of the resulting proof before it is published in its final citable form. Please note that during the production process errors may be discovered which could affect the content, and all legal disclaimers that apply to the journal pertain.
} 
chromatin as substrates, the transcriptional regulatory activity of RIP140 was validated, that altered its target chromatin structure [4]. Its physiological role was demonstrated in gene knockout studies [5], which revealed a functional role in differentiation/function of adipocyte and hepatocyte [6,7]. Further, at the gene level, a large number of genes were found to be abnormally expressed in RIP140 null mouse tissues [6,8], confirming its gene regulatory function.

Recently, our systemic mass spectrometry (MS) studies of RIP140 purified from insect cultures uncovered its extensive post-translational modifications (PTMs) in mammalian cells [1,912]. These PTMs not only altered the property and function of RIP140 but also triggered its specific sub-cellular translocation [12]. The biochemically established, and functionally validated, PTMs of RIP140 include phosphorylation [1,9], acetylation [11], methylation [12] and pyridoxal 5'-phosphate (PLP) conjugation [10]. Interestingly, while an in vitro acetylation assay using histone acetyltransferase (HAT)-containing p300 demonstrated acetylation of RIP140 on Lys ${ }^{446}$ [13], this particular PTM could not be found in our MS spectra of RIP140 purified from insect cultures. From our comprehensive studies of its PTM, it has become clear that the gene repressive activity of RIP140 is regulated, directly, by mitogen-activated protein kinase (MAPK)-triggered phosphorylation and lysine acetylation (both enhancing its repressive activity), and, indirectly, by arginine methylation (reducing its repressive activity by stimulating its nuclear export) and PLP conjugation (enhancing its repressive activity by increasing in its nuclear retention). Given that two forms of PTM, phosphorylation and acetylation, both could directly enhance its repressive activity, it was interesting whether and how these two types of PTMs might interact. Further, it was unclear what triggered lysine acetylation of RIP140 in vivo.

In this current study, we started with an interest in determining the signaling pathways regulating lysine acetylation of RIP140. As it turned out, lysine acetylation of RIP140 could occur via the HAT activity of p300 in vivo, which was facilitated by the preceding threonine phosphorylation on two specific residues and stimulated by a specific MAPK, extracellularsignal-related kinase 2 (Erk2) that was activated in differentiating adipocyte cultures. We now report the data 1) validating p300 as the specific acetyltransferase for Lys ${ }^{158}$ and Lys ${ }^{287}$ acetylation of RIP140 in vivo, that is critical for its gene repressive activity, 2) delineating signal transduction from Erk2-stimulated $\mathrm{Thr}^{202}$ and $\mathrm{Thr}^{207}$ phosphorylation to $\mathrm{p} 300$-triggered lysine acetylation of RIP140, 3) determining the mechanism that facilitates direct interaction of RIP140 with p300, and 4) evaluating the in vivo kinetics of the activation of this signal transduction pathway in regulating the biological activity of RIP140 in fat accumulation of adipocytes.

\section{Material and Methods}

\section{Cell culture and transfection}

COS- 1 cells were maintained at $37^{\circ} \mathrm{C}$ in a $\mathrm{CO}_{2}$ incubator in DMEM supplemented with $10 \%$ fetal bovine serum. 3T3-L1 fibroblasts were maintain in DMEM containing 10\% calf serum, and differentiated by a cocktail including insulin, triiodothyronine, dexamethasone and isobutylmethylxanthine [14]. siRNAs were introduced by Hiperfect (Qiagen, Valencia, CA, USA).

\section{Plasmid and mutagenesis}

Plasmids of Gal4-fused RIP140, Gal4-binding sites containing reporter, VP16-RIP140 and GST-RIP140 N-terminal domain were as described [15] pCI-p300 full length and pCI-p300 $\triangle$ HAT were gifts from Dr. Boyes [16]. RIP140 MAPK phosphorylation mutants were as 
previously described [1]. Mutagenesis was conducted by a Site-directed mutagenesis kit (Stratagene). Mutation primers were:

$$
\begin{aligned}
& \text { K111Q 5' -gtgaatttaaacgtacagaaggaagcgttgctg-3' } \\
& \text { K111A 5' -gtgaatttaaacgtagcgaaggaacgttgctg-3' } \\
& \text { K158Q 5'-attagacagagcctccaggagcagggatatgcc-3' } \\
& \text { K158A 5' -attagacagagcctcgcggagcagggatatgcc-3' } \\
& \text { K287Q 5'-cgggaacatgctctacaaacgcagaacgcacat-3' } \\
& \text { K287A 5'-cgggaacatgctctagcaacgcagaacgcacat-3' } \\
& \text { K311Q 5' 'caagagaatgggcagcaggacgtgggcagttcg-3' } \\
& \text { K311A 5'-caagagaatgggcaggcggacgtgggcagttcg-3' }
\end{aligned}
$$

\section{Reporter assay}

Luciferase reporter assay was conducted as described [1]. Briefly, COS-1 cells were culture in 24-well plates and transfected using Lipofectamine 2000 with 250ng of GAL4-TK-luc reporter, 50ng of SV40-LacZ internal control and 100ng of GAL4 fused RIP140 constructs. $48 \mathrm{hrs}$ post-transfection, cells were lysed and luciferase and LacZ activities were determined.

\section{Western blotting and immunoprecipitation}

To prepare whole cell lysates, cells were washed twice with cold PBS and harvested in RIPA buffer (50mM Tris-HCl pH 7.4, 0.5\% deoxycholic acid, 150mM NaCl, $0.1 \%$ SDS, 4Mm EDTA and $1 \%$ NP-40) with a protease inhibitor cocktail (Roche Applied Science, Indianapolis, IN, USA), $1 \mathrm{mM}$ PMSF, $1 \mathrm{mM}$ sodium fluoride and $1 \mathrm{mM}$ sodium orthovanadate. After centrifugation, supernatant was collected and protein concentrations were determined using Bradford method and subjected into SDS-PAGE. For immunoprecipitation, cell were lysed and collected with a buffer (50mM Tris-HCl pH 8.0, $10 \%$ glycerol, $100 \mathrm{mM} \mathrm{NaCl}, 1 \mathrm{Mm}$ EDTA and $0.1 \%$ NP-40) containing protease inhibitor cocktail, $1 \mathrm{mM} \mathrm{PMSF}, 1 \mathrm{mM}$ sodium fluoride and $1 \mathrm{mM}$ sodium orthovanadate for immunoprecipitation. Equal amount of proteins were incubate with primary antibodies overnight, and supplemented with protein $\mathrm{G}$ beads for another 1 hour. After five times washing by the IP buffer, proteins were eluted by boiling at $100^{\circ} \mathrm{C}$ for 5 mins in 2X Laemmeli loading buffer. Antibodies against Erk1, Erk2, phsopho-Erk1/2, phosphor-threonine and acetyl-lysine were purchase from Cell signaling. p300 antibody was purchased from Upstate. Flag and $\beta$-actin antibodies were purchased from Sigma and Santa Cruz, respectively. The levels of acetylated RIP140 with or without p300 (Fig. 5) were quantified using ImageJ, with the wild type RIP140 basal level (without p300) set as the value of 1 . Three sets of data were quantified. Statistics were obtained using student $t$ test.

\section{In vitro protein interaction assay}

In vitro protein interaction assay was performed as previously described method [17]. Briefly, $\left[{ }^{35}\right.$ S]methionine-labeled proteins were synthesized in vitro using TNT quick coupled transcription-translation system (Promega, Madison, WI, USA). Assays were performed in IP buffer at $4^{\circ} \mathrm{C}$ for 2 hours with gentle shaking. Immunocomplexes were incubated with antibody against p300 (Millipore, Billerica, MA, USA) at $4^{\circ} \mathrm{C}$ overnight and protein $\mathrm{G}$ beads for another 1 hours. After washing five times with the IP buffer, bound proteins were eluted in Laemmeli loading buffer and separated on a $6 \%$ SDS-PAGE which was dried and exposed to a phosphoimager screen (Molecular Dynamics). 


\section{Glycerol determination}

In the fat accumulation rescue experiment, RIP-null MEF cells were rescued by ectopically expressing wt or the indicated mutants. Quantification of fat accumulation was obtained by alkaline hydrolysis and measuring glycerol released using Free Glycerol Determination Kit (Sigma).

\section{Results \\ Lys $^{158}$ and Lys ${ }^{287}$ are required for p300-stimulated RIP140 trans-repressive activity}

By LC-ESI-MS, we previously identified 8 in vivo acetylated lysine residues of RIP140 purified from insect cultures [11], including four acetylated lysine residues in the amino-terminal domain, three in the central domain and one in the carboxyl-terminal domain. We further found that a deacetylase inhibitor, TSA, could enhance the trans-repressive activity, suggesting a potential role for acetylation in modulating the property and function of RIP140. We then determined that it was the amino-terminal domain of RIP140 that responded to the deacetylase inhibitor [11]. It was also reported that p300 acetyltransferase could acetylate RIP140 at $\mathrm{Lys}^{446}$ in vitro [13]. While we were able to confirm lysine acetylation in vivo, Lys ${ }^{446}$ acetylation was not found in our MS spectra of RIP140 purified from insect cultures [11]. To examine exactly if and how p300 affected lysine acetylation of RIP140 in vivo to regulate its activity, the effect of p300 on the trans-repressive activity of RIP140 was evaluated using a Gal4-reporter in COS-1 cells. As shown in Fig. 1A, p300 indeed enhanced the trans-repressive activity of RIP140 in vivo (columns 2 and 3); whereas the addition of p300 inhibitor, anacardic acid [18], reversed this effect and the inactive p300 (p300- $\Delta$ HAT) relieved RIP140's transrepressive activity (columns 4 and 5). The data confirmed that the trans-repressive activity of RIP140 indeed is regulated by the acetyltransferase activity of p300 in vivo.

It was suspected that p300 might be recruited to RIP140 for its acetylation. A mammalian twohybrid protein interaction assay was conducted to test this possibility. As shown in Fig. 1B, p300 interacted with the RIP140 full-length protein (AD-RIP140 FL) and its dissected aminoterminal domain (AD-RIP140 N) that was previously shown to be responsive to the deacetylase inhibitor. The carboxyl-terminal domain (AD-RIP140 C) was confirmed to lack the p300interacting property. Interestingly, this interaction was enhanced by a MAPK activator (MAPK Ac), and repressed by a MAPK inhibitor (MAPL In), indicating a potential cross-regulation of RIP140/p300 interaction by the MAPK pathway.

Within the amino-terminal domain of RIP140, four acetylated lysine residues were identified previously [11], including residues $111,158,287$ and 311 . We then mutated each residue individually to either an alanine (to abolish acetylation) or a glutamine (to mimic acetylation) $[19,20]$ and determined the responsiveness to p300-mediated trans-repressive activity using the same Gal4 reporter system in COS-1. It was suspected that mutating critical acetylated lysine into alanine (to abolish acetylation) would reduce its trans-repressive activity and p300 responsiveness; on the contrary, mutating the lysine residue into a glutamine (mimicking acetylation) would render RIP140 more repressive even without p300. As shown in Fig. 1C, among the four acetylated lysine residues, mutations on either one of the two residues, Lys ${ }^{158}$ and Lys ${ }^{287}$, resulted in the trans-repressive activity that partially met the criteria, suggesting that Lys ${ }^{158}$ and Lys $^{287}$ both were required, and possibly synergistically involved in lysine acetylation-enhanced trans-repressive activity of RIP140. We then generated double positive (K158/287Q, acetyl mimetic) and negative (K158/287A, blocking acetylation) mutants that were similarly tested in trans-repression assays. As shown in figure 1D, the acetylmimetic double mutant (K158/287Q) was strongly repressive with or without $\mathrm{p} 300$, whereas the double negative mutant (K158/287A) became very inefficient in trans-repression and was 
not significantly responsive to $\mathrm{p} 300$. All together, these results revealed that $\mathrm{p} 300$ regulated RIP140's trans-repressive activity may via, at least, acetylation on Lys ${ }^{158}$ and Lys ${ }^{287}$.

\section{Interdependence of p300 and MAPK-regulated trans-repressive activity of RIP140}

We have previously reported that phosphorylation of RIP140 by MAPK on $\mathrm{Thr}^{202}$ and $\mathrm{Thr}^{207}$ increased its trans-repressive activity, and then generated double negative (T202/207A) and double positive (T202/207E) mutants [1]. The fact that interaction of RIP140 with p300 could be modulated by MAPK (Fig. 1B) suggested an interesting possibility that MAPKtriggered phosphorylation and p300-mediated lysine acetylation of RIP140 might be crossregulated. To test this possibility, we examined the individual and combinatorial effects of inhibiting MAPK (to reduce threonine phosphorylation, rendering RIP140 less repressive) and inhibiting deacetylase (to enhance lysine acetylation, rendering RIP140 more repressive) on the trans-repressive activity of RIP140. As shown in Fig. 2A, in consistence with our previous finding, the MAPK inhibitor (MAPK In) reduced the trans-repressive activity of the wild type RIP140. The deacetylase inhibitor (Deacetylase In) rendered RIP140 strongly repressive; however which was effectively reversed by MAPK inhibitor. The effect of ERK1/2 inhibitor PD98059 was validated as shown on the right this figure. This result indicated that MAPKstimulated phosphorylation regulated the acetylation-stimulated trans-repressive activity of RIP140. This was further supported by the results using $\mathrm{Thr}^{202}$ and $\mathrm{Thr}^{207}$ double mutants. The phospho-mimetic RIP140 (T202/207E) was strongly repressive, which was not blocked by MAPK In. As predicted, the double de-phosphorylation mutant RIP140 (T202/207A) was less repressive, and it failed to respond to the enhancing effect of Deacetylase inhibitor. This result showed that dephosphorylation on $\mathrm{Thr}^{202}$ and $\mathrm{Thr}^{207}$ rendered RIP140 unresponsive to modulation by lysine acetylation, further supporting the regulation of RIP140 lysine acetylation by MAPK-triggered specific threonine phosphorylation.

We then examined the impact of MAPK activation on the enhancement of the trans-repressive activity of RIP140 by p300. As shown in figure 2B, MAPK activation further increased, whereas MAPK inhibition abrogated, the p300-enhanced trans-repressive activity of the wilt type RIP140. The phospho-mimetic mutant (T202/207E) was more repressive, and its activity was further enhanced by $\mathrm{p} 300$. As predicted, the phosphorylation negative mutant (T202/207A) was less repressive and failed to respond to $\mathrm{p} 300$ stimulation. These results confirmed that dephosphorylation on $\mathrm{Thr}^{202}$ and $\mathrm{Thr}^{207}$ rendered RIP140 unresponsive to modulation by p300-stimulated lysine acetylation, and also supported the notion of the regulation of RIP140 lysine acetylation by specific MAPK-triggered threonine phosphorylation.

\section{Erk2-dependent phosphorylation of RIP140 modulates its repressive activity}

Data shown above suggested regulation of lysine-acetylation on RIP140 by, presumably upstream, MAPK-mediated phosphorylation. In our preliminary studies of adipocyte differentiation system, Erk1 and Erk2 were both found to be expressed in differentiating cultures. We then first identified the kinase that regulated RIP140 phosphorylation and transrepressive activity by knocking down the endogenous Erk1 or Erk2 in COS-1 cells using siRNA, which were then used in trans-repressive assays. As shown in Fig. 3 top panel, MAPK Ac-enhanced trans-repressive activity of the wild type RIP140 was significantly reduced by Erk2 knockdown. To the phospho-mimetic mutant (T202/207E), knockdown of either Erk1 or Erk2 exerted no effect (middle panel) to RIP140 phospho-mimetic mutant's trans-repressive activity. The efficiency of Erk knockdown was evaluated by immunoblotting (bottom panel). This result revealed that Erk2 as the principal MAPK that phosphorylated RIP140 to enhance its trans-repressive activity. 


\section{RIP140 interaction with p300 is modulated by Erk2-mediated phosphorylation}

The facts that RIP140 interacted with p300 via its amino-terminal domain (Fig. 1B) and that MAPK (specifically Erk2) modulated p300-stimulated trans-repressive activity of RIP140 prompted us to examine whether RIP140 interaction with p300 could be regulated by Erk2triggered phosphorylation. We first conducted a mammalian two-hybrid assay to test the effect of phosphorylation on RIP140 interaction with p300 (Fig. 4A). Clearly, interaction of the amino-terminal domain of RIP140 (AD-EIP140 N) with p300 was enhanced by the phosphomimetic mutations (AD-RIP140 N-CP), but abrogated by the mutations blocking its phosphorylation (AD-RIP140 N-CN). We then conducted a direct in vitro protein interaction assay (Fig. 4B). The wilt type and phospho-mimetic mutant, but not the dephosphorylation mutant, effectively interacted with p300 and its enzymatically inactive mutant (p300- $\Delta$ HAT), supporting that RIP140 interaction with p300 could be enhanced by threonine phosphorylation on RIP140, and that acetyltransferase domain of p300 was not required for its interaction with RIP140.

We then used differentiated 3T3-L1 cells to verify immuno-complex formation of endogenous RIP140 with p300 in vivo. As shown in Fig. 4C, MAPK activation indeed enhanced the formation of RIP140/p300 complex, and Erk2 knockdown effectively reduced this complex formation. The same effect was observed for the ectopically expressed RIP140 and p300 in COS-1 cells (Fig. 4D) where RIP140 phospho-mimetic muatant (T202/207E), but not phosphorylation negative mutant (T202/207A), interacted with p300 despite its lacking the HAT activity. Therefore, the p300- $\triangle$ HAT mutant effectively interacted with RIP140, despite its failure to enhance the trans-repressive activity of RIP140 (Fig. 1A), supporting that the p300 HAT enzymatic activity was not required for its recruitment to RIP140 but was required for enhancing the trans-repressive activity of RIP140.

\section{MAPK-mediated phosphorylation of RIP140 promotes its lysine acetylation}

We next examined lysine acetylation status of RIP140 modulated by MAPK activity. As shown in Fig. 5A, MAPK Ac elevated the level of lysine acetylation of exogenous RIP140 in COS-1 cell. This was further confirmed by the significantly stimulating effect of p300 on the level of lysine acetylation of the wild type RIP140 or its phosphor-mimetic mutant (T202/207E), but not the dephosphorylated mutant (Fig. 5B). To directly demonstrate lysine acetylation of RIP140 by p300, in vivo acetylation assay was conducted (Fig. 5C) where the wild type, but not the K158/287Q or K158/287A mutant, could be detected with anti-acetyl-lysine antibody in the presence of $\mathrm{p} 300$, suggesting its acetylation on lysine residues by $\mathrm{p} 300$ in vivo. In agreement with figure 1D, these result indicated Lys-158 and 287 are the acetylation residues for p300-mediated acetylation and enhancement of RIP140's trans-repressive activity. The negative control, p300- $\triangle$ HAT mutant, failed to effectively acetylate the wild type RIP140, further confirming that RIP140 could be acetylated on lysine residues by p300 in vivo.

Taken together, these results confirmed that p300 acetylated RIP140, at least on Lys ${ }^{158}$ and Lys ${ }^{287}$, which was regulated by Erk2-mediated $\mathrm{Thr}^{202}$ and $\mathrm{Thr}^{207}$ phosphorylation.

\section{Kinetics of lysine acetylation and threonine phosphorylation of endogenous RIP140 in 3T3- L1 adipoctye differentiation}

To examine the physiological relevance of the signal transduction pathway initiated from threonine phosphorylation to lysine acetylation of RIP140, we examined the kinetics of modifications on endogenous RIP140, as well as the critical enzyme Erk2, in the 3T3-L1 differentiation model. Because RIP140 expression occurred on differentiation day 2 in 3T3L1 differentiation model (data not shown), we examine the RIP140 post-translational modification from day2. It appeared that the kinetics of lysine acetylation of RIP140 paralleled that of its threonine phosphorylation (Fig. 6A), both were strongly detected on day 3 . 
Consistently, Erk 2 phosphorylation (activation) became obvious on day 2 (Fig. 6B), slightly preceding threonine phosphorylation of RIP140. The p300 level remained relatively constant in later stages. The in vivo kinetic data supported the relevance of this signal transduction pathway initiated from Erk2 activation, leading to threonine phosphorylation of RIP140 and its subsequent lysine acetylation in the 3T3-L1 differentiation model.

\section{Effects of $\mathrm{Thr}^{202} / \mathrm{Thr}^{207}$ phosphorylation and Lys ${ }^{158} / \mathrm{Lys}^{287}$ acetylation on the biological activity of RIP140 in fat accumulation of differentiated adipocytes}

To further evaluate the physiological relevance of the signal transduction pathway of Erk2mediated phosphorylation and p300-mediated lysine acetylation of RIP140, we examined the trans-repressive activity of specifically mutated RIP140s, as well as their functional roles in modulating fat accumulation in differentiated adipocytes [5]. As shown in Fig. 7A, the transrepressive activity of the phospho-mimetic RIP140 (T202/207E), was strongly repressive, the quadruple mutant K158/287A-T202/207E (phospho-mimetic but deacetylated) and the dephosphorylated RIP140 (T202/207A) were less repressive, whereas the quadruple mutant K158/287Q-T202/207A (dephosphorylated but acetyl-mimetic) regained the repressive activity, supporting that while both threonine phosphorylation and lysine acetylation contributed to the repressive activity of RIP140, threonine phosphorylation appeared to be the upstream signal eliciting this pathway and lysine acetylation was the ultimate stimulus for its trans-repressive activity.

The functional role of RIP140 threonine phosphorylation and lysine acetylation in modulating fat accumulation was tested in rescue experiments using the established RIP140-null adipoctye differentiation model [8]. As shown in Fig. 7B, the wild type and the phospho-mimetic T202/207E mutant effectively rescued the defect in fat accumulation, whereas the phosphomimetic but de-acetylated mutant (K158/287A-T202/207E) was not as effective in rescuing the defect in fat accumulation of RIP140 null cultures. This result confirmed that acetylation on Lys ${ }^{158}$ and Lys ${ }^{287}$, stimulated by phosphorylation on $\mathrm{Thr}^{202}$ and $\mathrm{Thr}^{207}$ of RIP140, enhanced the biological activity of RIP140 for both gene trans-repression and fat accumulation in differentiated adipocytes.

\section{Discussion}

The functional role for RIP140 in modulating various biological processes has been demonstrated in a large number of studies [3,5]. Recent comprehensive MS mapping of its PTMs such as phosphorylation, acetylation, methylation and PLP conjugation has begun to shed light on why RIP140 could exert such a wide spectrum of biological activities in different experimental systems. The next logical questions would be what signal inputs stimulated these PTMs and whether and how they were regulated. The goal of this study was to address this exact question by uncovering the first cellular signal and signal transduction pathway stimulating specific biochemical alternations (threonine phosphorylation followed by lysine acetylation) of RIP140 that enhanced its gene-repressive activity in the established adipocyte differentiation model. The signaling pathway was delineated (Fig. 7C) that started with the activated Erk2 which phosphorylated two specific threonine residues of RIP40, thereby enhancing the recruitment of the second enzyme p300 to RIP140. The action of p300 resulted in increased lysine acetylation of RIP140 (Fig. 4 and Fig. 5) whose gene-repressive activity was then enhanced by the recruitment of the repressive enzyme machinery containing HDACs as we reported earlier (11).

We demonstrated the synergistic effect of acetylation on two specific residues, Lys ${ }^{158}$ and Lys ${ }^{287}$; however, RIP140 could be acetylated on at least 8 lysine residues, and possibly by other enzymes containing acetyl transferase activity in addition to $\mathrm{p} 300$. The signal inputs, as well as regulation, of acetylation on other lysine residues remain to be determined. While p300 
was shown to acetylate Lys ${ }^{446}$ of RIP140 in vitro, this could not be validated in our MS spectra of RIP140 purified from eukaryotic cells [11]. Therefore, it remains to be validated whether other lysine residues (in addition to Lys ${ }^{158}$ and Lys ${ }^{287}$ ) could be acetylated by $\mathrm{p} 300$ in vivo. Given the complicated and presumably dynamic changes of cellular factors in differentiating adipocytes, and there appeared to be other PTMs, such as arginine methylation and PLP conjugation, that regulated sub-cellular translocation of RIP140 (altering its nuclearcytoplasmic distribution) and therefore also affected its gene repressive activity [1,9-12], it would also be important to address whether and how these other forms of PTM might integrate and coordinate the biological activity of RIP140.

Other studies showed that reduction of either p300 or CBP (HAT-containing enzymes) suppressed adipogenesis [21] and that acetylation of certain transcription factors such as $\mathrm{C} /$ EBP $\beta$ and Foxo1 could regulate their activities in adipocyte differentiation [22,23]. Further, treatment with diallyl disulfide, an inhibitor of HDAC (thereby also enhancing protein acetylation), accelerated 3T3-L1 adipoctye differentiation and fat accumulation [24]. All the evidence indicated that protein acetylation was indispensable in adipocyte differentiation, and predicted that cellular environment for the regulation of protein acetylation in differentiating adipocyte cultures must be tightly controlled or coordinated. Our study was the first to delineate the signaling pathways that controlled protein acetylation of RIP140 in the adipoctye differentiation system. It is important to determine, in the future, how this signaling pathway in differentiating adipocytes may also regulate PTMs of other regulatory molecules that must be coordinated, or integrated, for the optimal differentiation efficiency and the function of fully differentiated adipocytes.

Sequential activation of transcriptional cascades during adipogenesis could be modulated by alteration in both intracellular and extracellular conditions. Erk activation has been shown to be triggered by the action of insulin in the early differentiating cells, mostly pre-adipocytes $[25,26]$. Erk1 is known to modulate adipogenesis, as illustrated in studies of knockout mice [27]; however, the functional role of Erk2 activity in adipogenesis was not clear. Our result showed that the effect of MAPK activation on RIP140 could be more significantly blocked by knockdown of Erk2, and Erk2 was critical to the recruitment of p300 to RIP140 (Fig. 4C). These results revealed an important role for Erk2 in regulating adipoctye differentiation, at least through its action on RIP140.

Crosstalk among different PTMs can be critical to the integration of diverse signaling pathways in a given biological system [28-30]. Further, most PTMs are reversible. The enzyme machineries for protein acetylation have begun to be discovered. While most of these enzymes were validated, initially, by using histone proteins as the substrates, increasingly, it has become clear that many of these enzymes in fact could also act on non-histone proteins. Our results showed the stimulation of p300-triggered lysine acetylation of RIP140 by its specific phosphorylation. However, we have not investigated the control of its deacetylation. Interestingly, it has long been reported that RIP140 could constitutively interact with various HDACs [31], which appeared to be the principal mechanism underlying its gene-repressive activity and could be enhanced by lysine acetylation of RIP140 [11]. While HDACs recruited to RIP140 could function to modify histone proteins on the target chromatin as previously demonstrated $(4,29)$, it is possible that some of these HDACs recruited by RIP140 may also regulate deacetylation of RIP140 itself. This is also an important question to be addressed in terms of the dynamics of acetylation/deacetylation of RIP140 in differentiating and differentiated adipocytes. Further, deacetylation and ubiquitination on lysine residues were thought to compete with each other for the regulation of protein activity and stability [3234]. While we have not determined the sites of ubiquitination on RIP140, it was clear to us that RIP140 could be ubiquitinated (data not shown). This also presents another challenging question for future investigation. 


\section{Abbreviations}

ERK1/2, extracellular-signal-related kinase 1/2; HAT, histone acetyltrransferase; HDAC, histone deacetylase; MAPK, mitogen-activated protein kinase; MS, mass spectrometry; PLP, pyridoxal 5'-phosphate; PTM, post-translational modification; RIP140, receptor interacting protein 140.

\section{Acknowledgement}

This work was supported, in part by Philip Morris USA Inc. and Philip Morris International, and by NIH grants DK54733, DK60521, K02-DA13926 and DA11190 to L.-N. Wei. We thank Dr. Boyes for providing pCI-p300 $\Delta$ HAT and Dr. M. Parker for RIP140 null MEFs. We thank technical support from Y-W Lin.

\section{Reference}

1. Gupta P, Huq MD, Khan SA, Tsai NP, Wei LN. Mol Cell Proteomics 2005;4(11):1776-1784. [PubMed: 16093479]

2. L'Horset F, Dauvois S, Heery DM, Cavailles V, Parker MG. Mol Cell Biol 1996;16(11):6029-6036. [PubMed: 8887632]

3. Christian M, White R, Parker MG. Trends Endocrinol Metab 2006;17(6):243-250. [PubMed: 16815031]

4. Hu X, Chen Y, Farooqui M, Thomas MC, Chiang CM, Wei LN. J Biol Chem 2004;279(1):319-325. [PubMed: 14581481]

5. Steel JH, White R, Parker MG. J Endocrinol 2005;185(1):1-9. [PubMed: 15817822]

6. Leonardsson G, Steel JH, Christian M, Pocock V, Milligan S, Bell J, So PW, Medina-Gomez G, VidalPuig A, White R, Parker MG. Proc Natl Acad Sci U S A 2004;101(22):8437-8442. [PubMed: 15155905]

7. White R, Morganstein D, Christian M, Seth A, Herzog B, Parker MG. FEBS Lett 2008;582(1):39-45. [PubMed: 18023280]

8. Christian M, Kiskinis E, Debevec D, Leonardsson G, White R, Parker MG. Mol Cell Biol 2005;25 (21):9383-9391. [PubMed: 16227589]

9. Huq MD, Khan SA, Park SW, Wei LN. Proteomics 2005;5(8):2157-2166. [PubMed: 15846843]

10. Huq MD, Tsai NP, Lin YP, Higgins L, Wei LN. Nat Chem Biol 2007;3(3):161-165. [PubMed: 17277785]

11. Huq MD, Wei LN. Mol Cell Proteomics 2005;4(7):975-983. [PubMed: 15879431]

12. Huq, Mostaqul MD.; Gupta, P.; Tsai, NP.; White, R.; Parker, MG.; Wei, LN. Embo J 2006;25(21): 5094-5104. [PubMed: 17053781]

13. Vo N, Fjeld C, Goodman RH. Mol Cell Biol 2001;21(18):6181-6188. [PubMed: 11509661]

14. Bogan JS, McKee AE, Lodish HF. Mol Cell Biol 2001;21(14):4785-4806. [PubMed: 11416153]

15. Chuang FM, West BL, Baxter JD, Schaufele F. Mol Endocrinol 1997;11(9):1332-1341. [PubMed: 9259323]

16. Boyes J, Byfield P, Nakatani Y, Ogryzko V. Nature 1998;396(6711):594-598. [PubMed: 9859997]

17. Guidez F, Howell L, Isalan M, Cebrat M, Alani RM, Ivins S, Hormaeche I, McConnell MJ, Pierce S, Cole PA, Licht J, Zelent A. Mol Cell Biol 2005;25(13):5552-5566. [PubMed: 15964811]

18. Balasubramanyam K, Swaminathan V, Ranganathan A, Kundu TK. J Biol Chem 2003;278(21): 19134-19140. [PubMed: 12624111]

19. Shimizu R, Trainor CD, Nishikawa K, Kobayashi M, Ohneda K, Yamamoto M. J Biol Chem 2007;282 (21):15862-15871. [PubMed: 17374603]

20. Thomas M, Dadgar N, Aphale A, Harrell JM, Kunkel R, Pratt WB, Lieberman AP. J Biol Chem 2004;279(9):8389-8395. [PubMed: 14670946]

21. Takahashi N, Kawada T, Yamamoto T, Goto T, Taimatsu A, Aoki N, Kawasaki H, Taira K, Yokoyama KK, Kamei Y, Fushiki T. J Biol Chem 2002;277(19):16906-16912. [PubMed: 11884404]

22. Jing E, Gesta S, Kahn CR. Cell Metab 2007;6(2):105-114. [PubMed: 17681146] 
23. Wiper-Bergeron N, Salem HA, Tomlinson JJ, Wu D, Hache RJ. Proc Natl Acad Sci U S A 2007;104 (8):2703-2708. [PubMed: 17301242]

24. Lee JH, Kim KA, Kwon KB, Kim EK, Lee YR, Song MY, Koo JH, Ka SO, Park JW, Park BH. Int J Mol Med 2007;20(1):59-64. [PubMed: 17549389]

25. Bost F, Aouadi M, Caron L, Binetruy B. Biochimie 2005;87(1):51-56. [PubMed: 15733737]

26. Rosen ED, MacDougald OA. Nat Rev Mol Cell Biol 2006;7(12):885-896. [PubMed: 17139329]

27. Bost F, Aouadi M, Caron L, Even P, Belmonte N, Prot M, Dani C, Hofman P, Pages G, Pouyssegur J, Le Marchand-Brustel Y, Binetruy B. Diabetes 2005;54(2):402-411. [PubMed: 15677498]

28. Henikoff S. Nat Rev Genet 2008;9(1):15-26. [PubMed: 18059368]

29. Vervoorts J, Luscher-Firzlaff J, Luscher B. J Biol Chem 2006;281(46):34725-34729. [PubMed: 16987807]

30. Yang XJ. Oncogene 2005;24(10):1653-1662. [PubMed: 15744326]

31. Wei LN, Hu X, Chandra D, Seto E, Farooqui M. J Biol Chem 2000;275(52):40782-40787. [PubMed: 11006275]

32. Feng L, Lin T, Uranishi H, Gu W, Xu Y. Mol Cell Biol 2005;25(13):5389-5395. [PubMed: 15964796]

33. Li X, Zhang S, Blander G, Tse JG, Krieger M, Guarente L. Mol Cell 2007;28(1):91-106. [PubMed: 17936707]

34. Perkins ND. Oncogene 2006;25(51):6717-6730. [PubMed: 17072324] 
(A)

\section{Enhancement of RIP140 trans-repressive function by $\mathrm{p} 300$ activity}

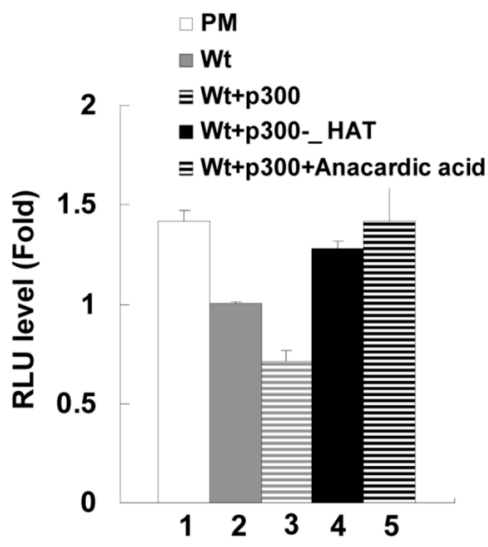

(C)

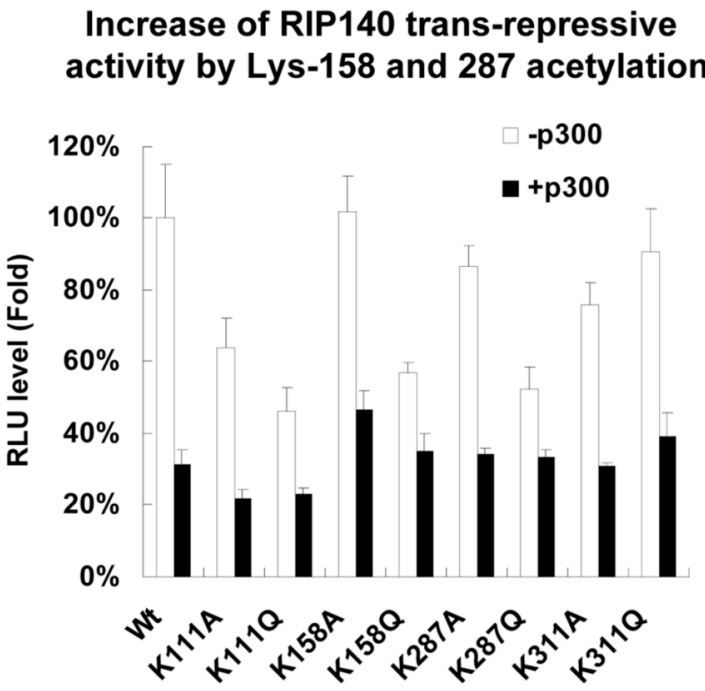

(B)

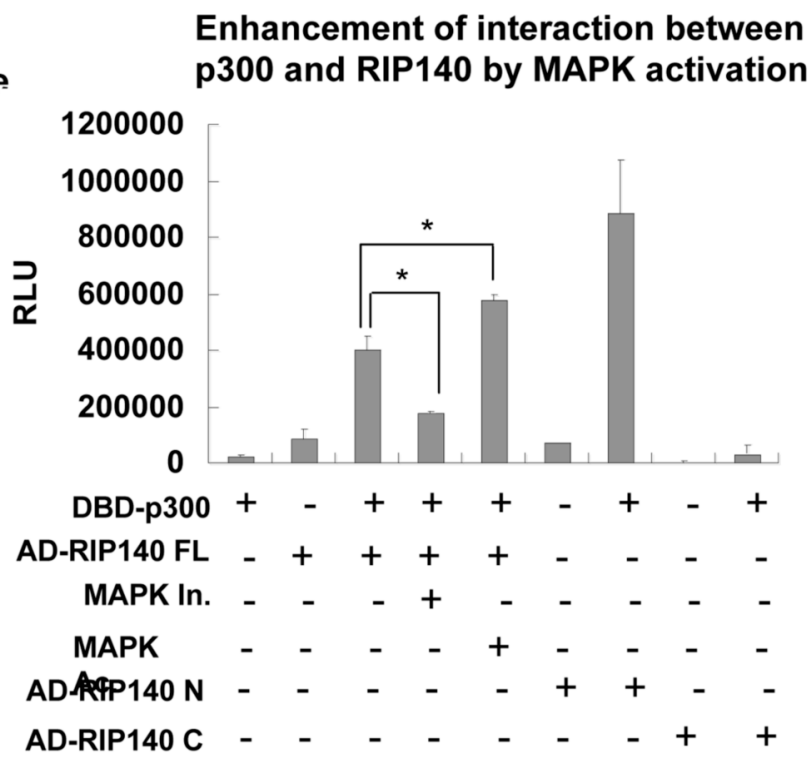

(D)

Effect of Lys-158 and 287 double mutations in p300-regulated RIP140 trans-repressive activity

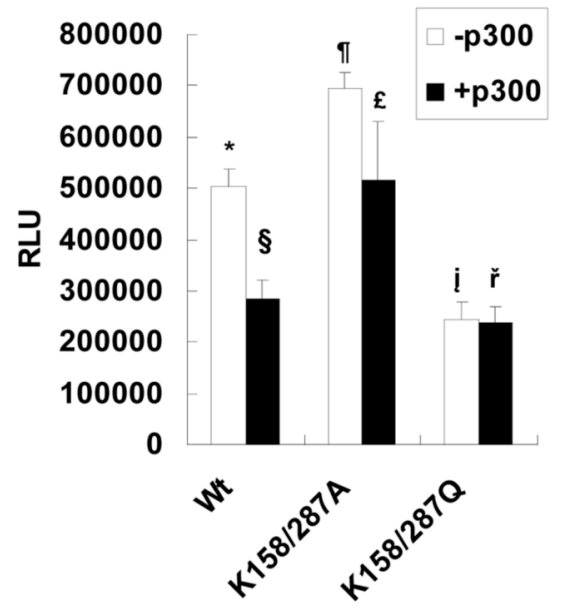

Figure 1. Requirement for $\mathrm{Lys}^{\mathbf{1 5 8}}$ and $\mathrm{Lys}^{287}$ in p300-enhanced trans-repressive activity of RIP140 (A) Trans-repressive activities of wild type RIP140 were compared in the presence of p300, p300- $\triangle$ HAT or a p300 inhibitor, anacardic acid, in COS-1 cells. Statistics: lane 3 compared with lane $2(p<0.01)$; lane 4 versus lane $2(p<0.005)$; lane 5 versus lane $3(p<0.03)$. (B) Interaction of $\mathrm{p} 300$ and RIP140 were monitored in COS-1 cells by mammalian two-hybrid assay. Cells were treated with MAPK activator (MAPK Ac, anisomycin) or MAPK inhibitor (MAPK In, PD98059) in DMEM containing 10\%FBS for 8hrs. *: $p$ value $<0.02$. (C) Transrepressive activity of indicated lysine mutation in the amino-terminal domain of RIP140 in the absence or presence of p300. (D) Trans-repressive activity of RIP140 and acetylation mutants, 
K158/287Q and K158/287A in the presence or absence of p300 in COS-1 cells. Statistics: * versus $\S(p$ value $=0.02)$; đI versus $£(p$ value $=0.25)$; i versus $\check{r}(p$ value $=0.9$. 


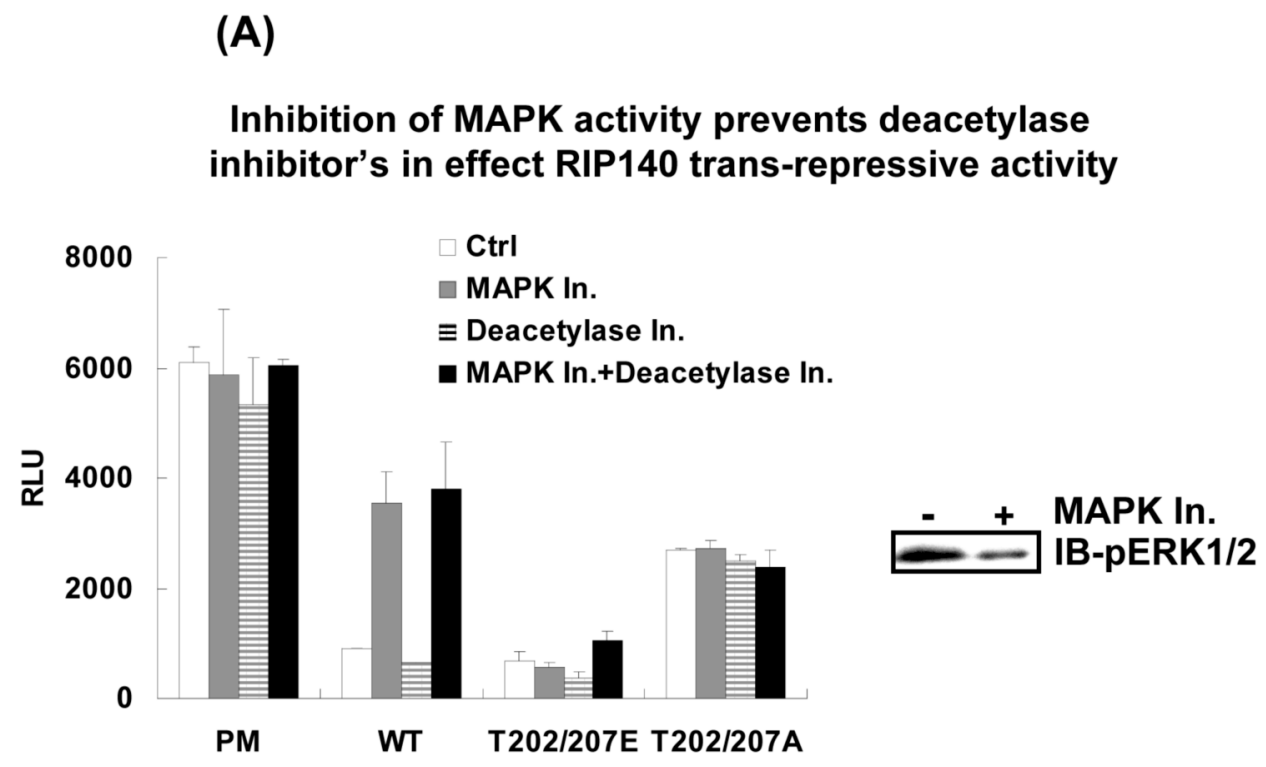

(B)

\section{Reduction in p300-mediated RIP140's trans-repressive activity by Thr-202 and 207 phosphorylation}

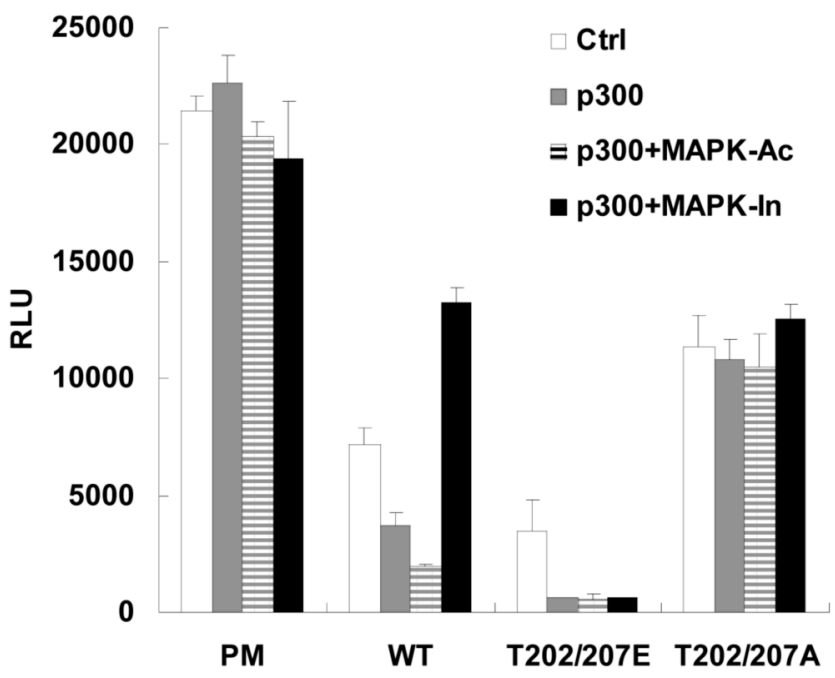

Figure 2. Effects of MAPK activation on p300-enhanced trans-repressive activity of RIP140 (A) Trans-repressive activities of RIP140 wild type and phosphorylation mutants (T202/207E and T202/207A) affected by MAPK inhibitor (MAPK In, PD98059) and deacetylase inhibitor (Deacetylase In, TSA) in COS-1. The effect of PD98059 was validated as shown on the right. (B) COS-1 cells were transfected with control vector (PM) or indicated Gal4DBD-fused RIP140 constructs with or without p300. After $40 \mathrm{hrs,} \mathrm{cells} \mathrm{were} \mathrm{treated} \mathrm{with} \mathrm{indicated}$ treatment or DMSO as control (Ctrl) for another 8hrs. Trans-repressive activities of RIP140 wild type and phosphorylation mutants (T202/207E and T202/207A) were determined by luciferase activities. 


\section{Reduction of RIP140 trans-repressive activity by Erk2 knockdown}

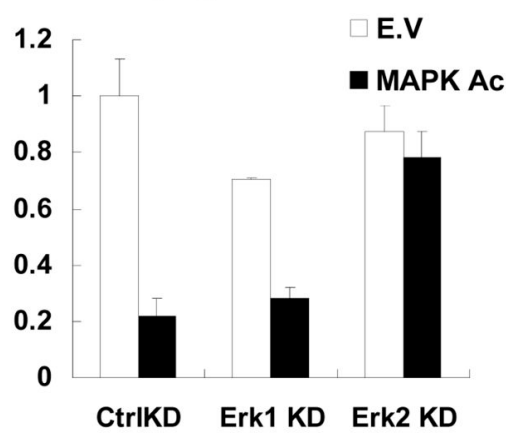

\section{Trans-repressive assay of RIP140-T202/207E mutant}

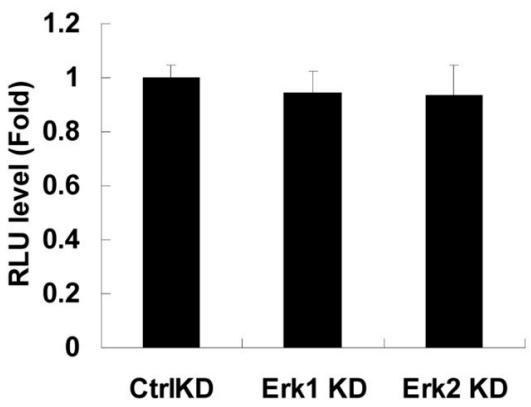

Erk1/2 knockdown efficiency

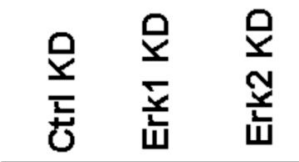

IB-Erk1 IB-Erk2 IB-actin

Figure 3. Erk2-dependent threonine phosphorylation and trans-repressive activity of RIP140 Upper panel: trans-repressive activities of wild type RIP140 in the absence or presence of MAPK activator in COS-1 cells transfected with Erk1 or Erk2 siRNAs, respectively. Middle panel: trans-repressive activity of RIP140 phospho-mimetic mutant, RIP140-T202/207E, in the presence of MAPK activator in COS-1 cells transfected with Erk1 or Erk2 siRNA, respectively. Lower panel: the knockdown efficiencies of Erk1 and Erk2 were monitored by western blotting. Actin is a loading control. 
(A)

(C)

Reduction of interaction between RIP140 and $\mathrm{p} 300$ by Erk2 knockdown

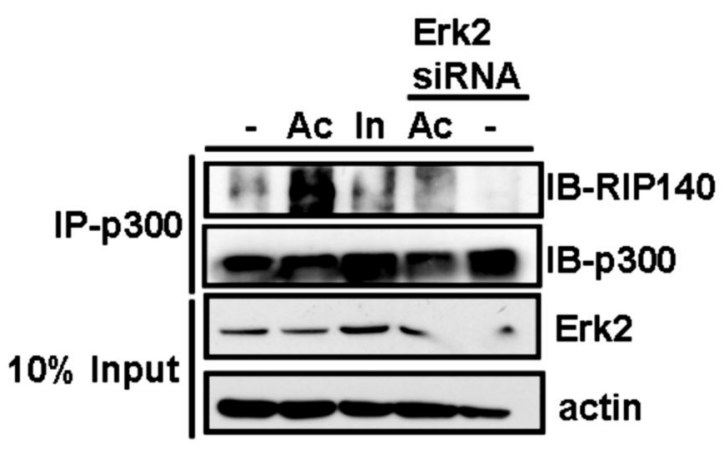
phosphorylation on T202/207
(B)

\section{Increase of interaction between p300 and \\ RIP140 by T202/207 phosphorylation}

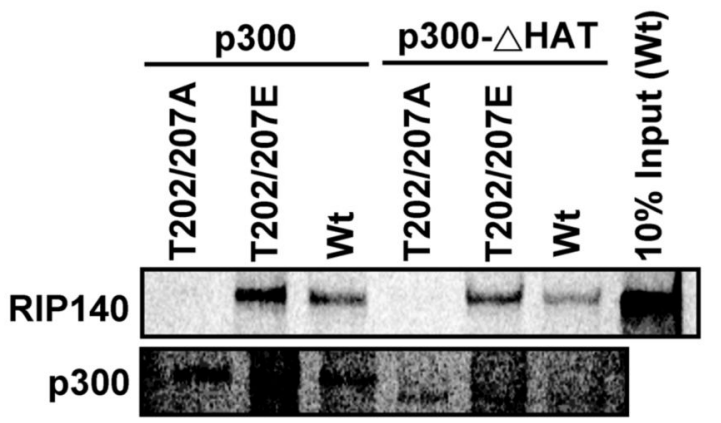

(D)

Co-IP of exogenous RIP140 and p300

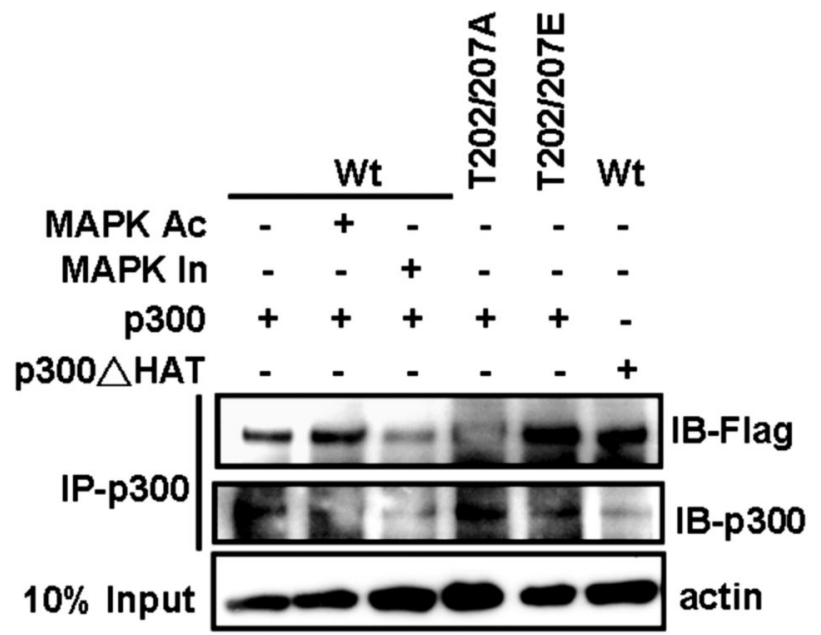

Figure 4. Regulation of interaction between p300 and RIP140 by Erk2-mediated RIP140

(A) Mammalian two-hybrid assay for p300 and RIP140 aminal terminus was conducted in COS-1 cells. AD-RIP140 N: the amino-terminal domain of RIP140 fused to the AD domain of Gal4. AD-RIP140 N-CP: the phospho-mimetic (T202/207E) mutant RIP140 amino terminal domain fused to the AD domain of Gal4; AD-RIP140 N-CN (T202/207A), the phosphorylation negative mutant RIP140 amino terminal domain fused to the AD domain of Gal4. *: $p$ value $<0.02$; **: $p$ value $<0.0002$. (B) In vitro protein interaction assay of $\mathrm{p} 300$, or its enzymatically inactive counterpart, p300- $\triangle$ HAT, with RIP140 wild type (WT), or its phosphorylation mutants T202/207E and T202/207A. Assay was performed as described in 
materials and methods part. Immunocomplexes were immunoprecipitated by anti-p300 antibody. (C) In vivo complex formation of endogenous p300 and RIP140 in differentiated 3T3-L1 affected by MAPK Ac, MAPK In, or with additional ERK2 siRNA. 3T3-L1 cells were differentiated by differentiation medium. siRNAs were transfected into differentiating cells on day 2. On day 4 of differentiation, cells were treated with MAPK activator, MAPK inhibitor or DMSO as control for $8 \mathrm{hrs}$. Cell lysates were collected and subjected into Co-IP and western blotting. (D) Complex formation of exogenously provided p300 wild type or mutant, and RIP140 wild type or mutants, in COS-1 cells. Cells were co-transfected flag-RIP140 and its mutants (T202/207A and T202/207E) with p300 or its enzyme-defective mutant for $40 \mathrm{hrs}$. Cells were then treated with indicated drugs for another 8 hrs. Interaction of p300 and flagRIP140 was monitored by probing the anti-p300-precipitated complex with anti-flag. 
(B)

\section{T202/207 phosphorylation regulates p300-modulated lysine acetylation}

(A)

Lysine acetylation of exogenous Flag-RIP140
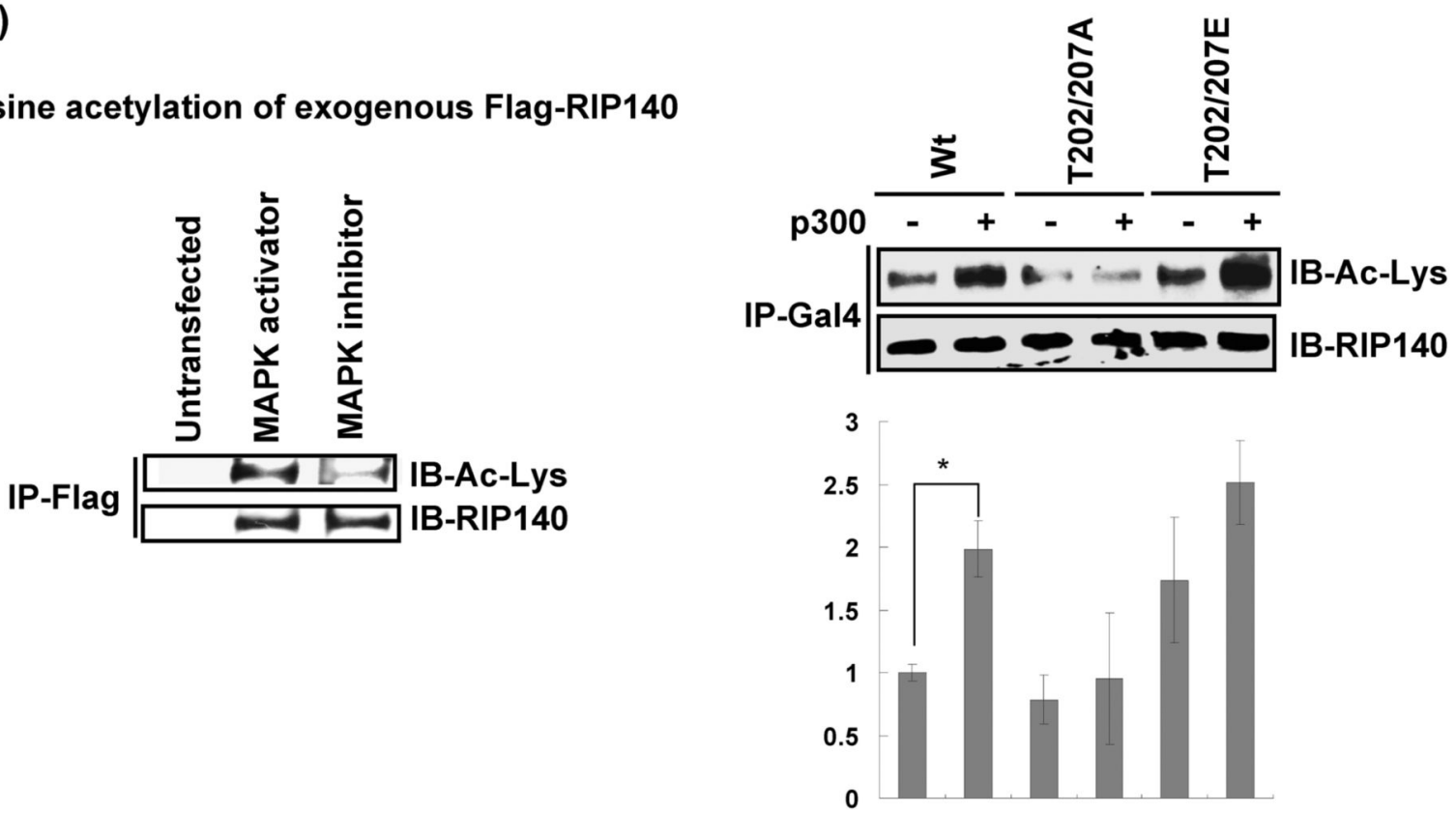

(C)

Decrease of p300-mediated lysine acetylation by mutations in K158 and K287

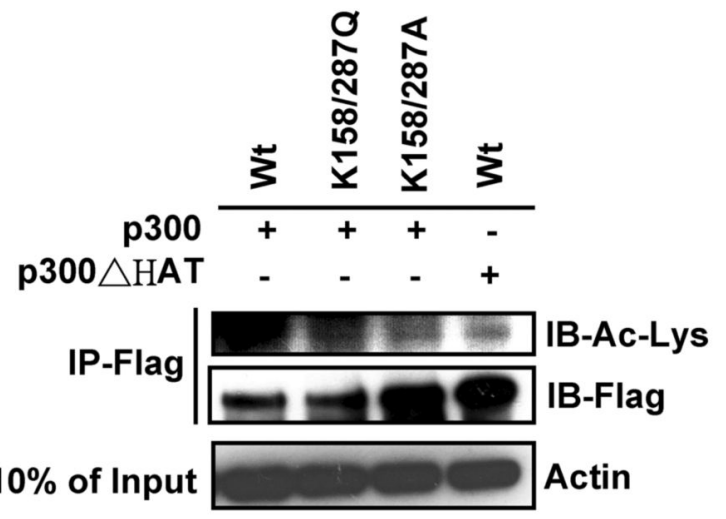

Figure 5. Modulation of p300-mediated K158 and K287 acetylation of RIP140 by MAPK (A) Lysine acetylation of RIP140 affected by MAPK in COS-1 cells. Cells were transfected with flag-RIP140 for $40 \mathrm{hrs}$ and then with MAPK activator $(1 \mu \mathrm{M})$ or MAPK inhibitor $(3 \mu \mathrm{M})$ for another $8 \mathrm{hrs}$. Anti-flag immunoprecipitates were monitored with anti-acetyl-lysine and anti-RIP140 antibodies. (B) Lysine acetylation of RIP140 affected by p300 and phosphorylation on $\mathrm{Thr}^{202} / \mathrm{Thr}^{207}$ in COS-1 cells. Cells were co-transfected Gal4DBD-fused RIP140 wild type or mutants (T202/207A and T202/207E) with or without p300 for 48 hrs. Anti-Gal4 immunoprecipitates were monitored with anti-acetyl-lysine and anti-RIP140 antibodies. The levels of acetylated RIP140 were quantified using ImageJ. The basal level of wild type RIP140 (without p300 co-transfection) was arbitrarily set as the value of 1, and 
statistical analysis of the wild type RIP140 with or without $\mathrm{p} 300$ was performed using three sets of data. *: $p<0.03$. (C) In vivo acetylation of Flag-RIP140 and its mutants by p300 in COS-1 cells. Cells were co-transfected flag-RIP140 and mutants (K158/287Q and K158/287A) with p300 or p300 enzyme defective mutant (p300 $\Delta$ HAT) for $48 \mathrm{hrs}$. Lysine acetylation was monitored by probing with anti-acetyl-lysine antibody for anti-flag immnuoprecipitates. 


\section{(A)}

\section{Kinetics of threonine phosphorylaiton and lysine acetylation of endogenous RIP140 in differentiating 3T3-L1}

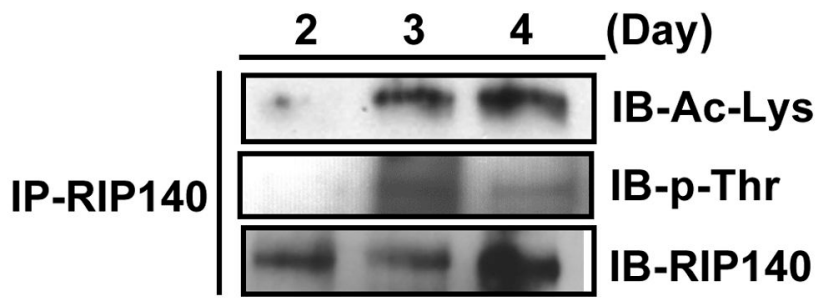

(B)

\section{Kinetics of endogenous enzyme activation in differentiating 3 T3-L1}

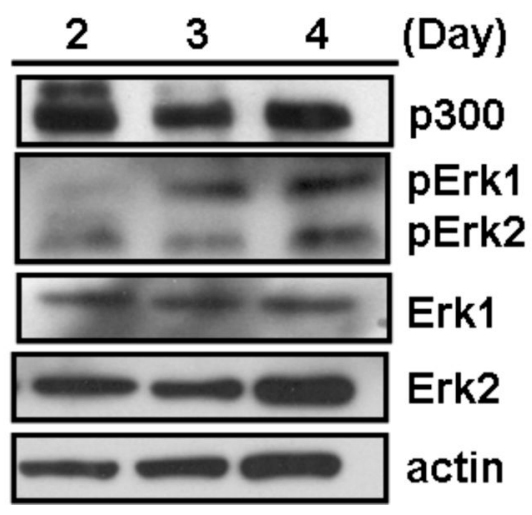

Figure 6. Kinetics of lysine acetylation and threonine phosphorylation of endogenous RIP140 and Erk1/2 activation in differentiating 3T3-L1

(A) Lysine acetylation and threonine phosphorylation of endogenous RIP140 in early differentiating 3T3-L1. Cell lysates of 3T3-L1 at indicated differentiation time point were collected. Acetylated lysine level and phosphorylated threonine level were monitored by probing anti-RIP140 immunoprecipitates with specific antibodies. (B) Expression and activation of endogenous ERKs in early differentiating 3T3-L1. 
(A)

Trans-repressive assay

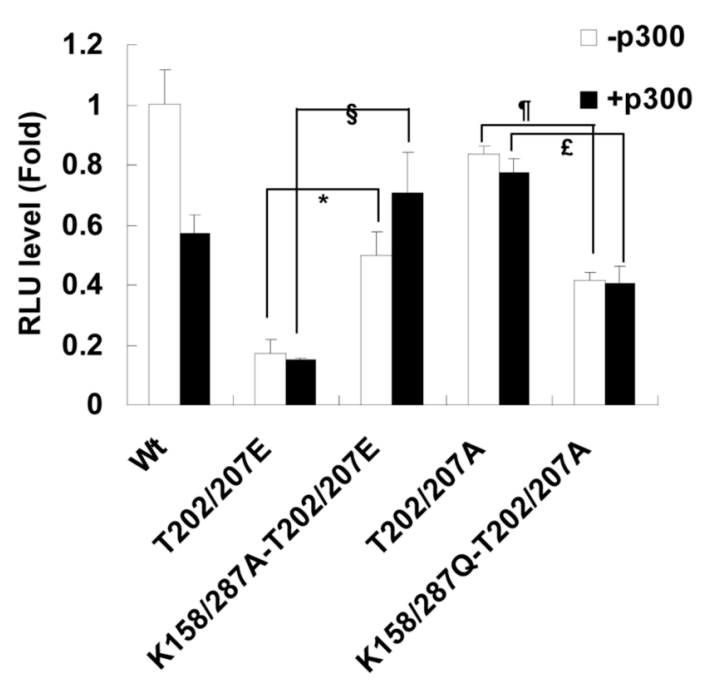

(B)

Decreased fat accumulation by expression of acetylation-defective RIP140

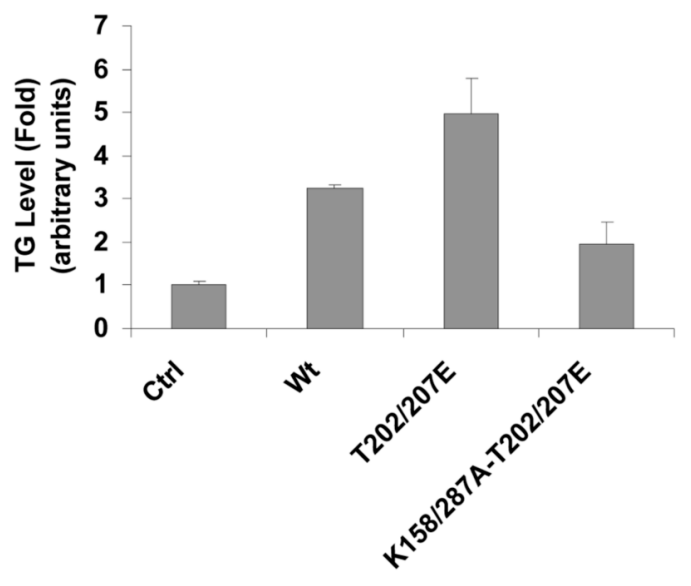

(C)

Signaling pathway of threonine phosphorylation/lysine acetylation that enhances RIP140's gene repressive activity

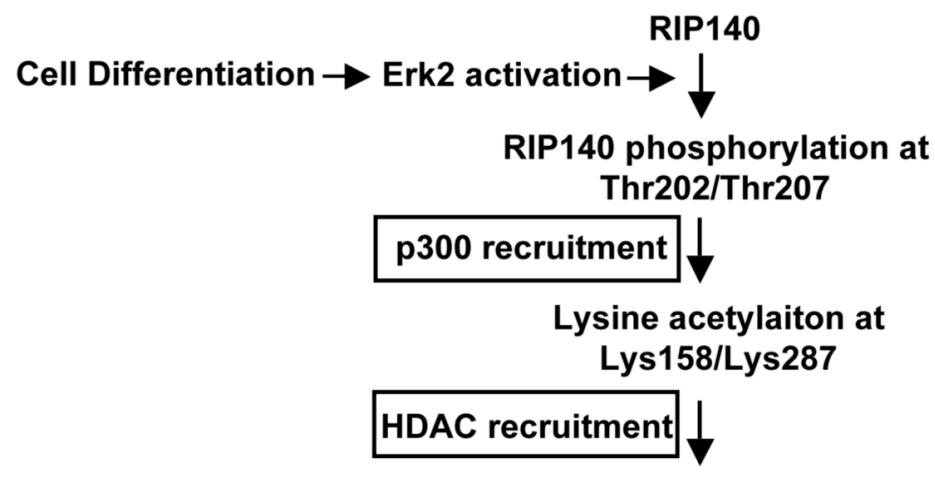

Enhanced repressive activity of RIP140

Figure 7. Biological activity of Lys ${ }^{158} / \mathrm{Lys}^{287}$ acetylation and $\mathrm{Thr}^{202} / \mathrm{Thr}^{207}$ phosphorylation of RIP140 in trans-repression and fat accumulation

(A) Trans-repressive activity of RIP140 wild type and mutants in COS-1 cells. *: $p$ value $=0.004 ; \S: p$ value $=0.05$; II: $\mathrm{p}$ value $=0.007 ; £: p$ value $=0.02)(\mathrm{B})$ Rescue of defect in fat accumulation in RIP140-null MEF cells by RIP140 wild type and mutants. RIP140-null MEF cells were differentiated following differentiated protocol. Transfection was performed on day 3 and day6. TG levels were determined on day 8. (C) A proposed signal transduction pathway, from Erk2-triggered threonine phosphorylation to p300-mediated lysine acetylation of RIP140. 